\title{
Application analysis on floating start of ESGM on the Sea Area
}

\author{
Yan Jun, Liu Zhi
}

China Satellite Maritime Tracking and Control Department, Jiangyin, 214431, China

Keywords: TT\&C ship; ESGM; low latitude; six calibration; floating

\begin{abstract}
The electrostatic gyro monitor(ESGM) has complicated structure and needs a long time to start up ,it requires sailing at a uniform course and casting fin to reduce ship shake ,avoiding sailing at low latitude $\left(6^{\circ} \mathrm{N} \sim 6^{\circ} \mathrm{S}\right)$, which is difficult to achieve cause of the limitation of mission area and weather condition,in order to solve this problem ,this paper provides a new method to start up ESGM while the ship is floating on the ocean,by analyzing the srating principle of ESGM and the data of the test,the result shows that ESGM can be well implemented in floating start , and it also provides a reference for different ship in using ESGM at sea.
\end{abstract}

\section{Introduction}

ESGM has the advantages of high precision, high stability and high reliability. The static bearing supports the mechanical support of gyro drift. The gyro drift accuracy can reach $0.0001 \circ / \mathrm{h}$, and it can output high accuracy position and heading information. Supporting the use of the composition of ESGM / INS combined system can significantly improve the navigation accuracy and extend the use of external information INS reset cycle. At present, the electro-static gyro monitor has been widely used in aerospace TT \& C ship, which has played an important role in ensuring TT \& C ship ship position measurement precision. In recent years, TT \& Low-latitude drifting start can effectively improve the probability of the successful start of the static gyro monitor, but also to avoid a wide range of maneuvering the ship, saving fuel, maximizing the service life of equipment, to enhance the TT \& C ship mission capability is important and urgent demand.

\section{Static start running characteristics}

The operation of the static gyro monitor (shown in Figure 1) is divided into the follow-up and gyro alignment of the gantry, gyro start (gyro start, steady speed, follow-up), initial alignment School "), 48 hours calibration, navigation.

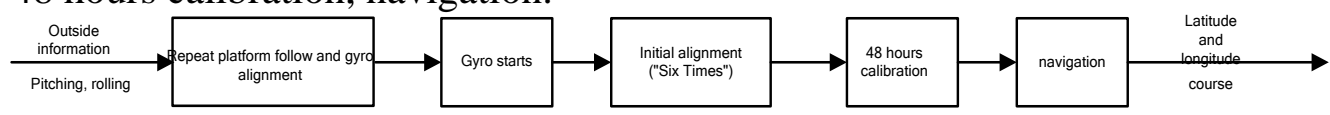

Figure 1. Starting Process of Electrostatic

\section{1 "Six times school"}

In the "sixth school" stage, the initial coordinates of the H-axis of the gyro are calculated by iteratively resolving the instantaneous local time angle, the instantaneous declination and the Greenwich time angle.

$$
\delta_{i}(0)\left\{\begin{array}{l}
S_{i}^{p}(\mathrm{t})=S_{i}(0)+\Delta S_{i}^{\omega_{e}}(\mathrm{t})+\Delta S_{i}^{C}(\mathrm{t}) \\
\delta_{i}^{p}(\mathrm{t})=\delta_{i}(0)+\Delta \delta_{i}^{\omega_{e}}(\mathrm{t})+\Delta \delta_{i}^{C}(\mathrm{t}) \\
S_{i}^{* p}(\mathrm{t})=S_{i}^{p}(\mathrm{t})+\lambda(\mathrm{t})
\end{array}\right.
$$

The height angle and azimuth angle measured by the gyroscope in the horizontal coordinate system are obtained by the spherical triangular transformation. The position angle of the gyro dynamic distance axis in the equatorial coordinate system is as follows:

$$
\left\{\begin{array}{l}
\cos h_{i} \cdot \sin A=-\cos \delta_{i} \cdot \sin S_{i}^{*} \\
\sinh h_{i}=\sin \delta_{i} \sin \varphi+\cos \delta_{i} \cdot \cos \varphi \cdot \cos S_{i}^{*} \\
\cos h_{i} \cdot \cos A_{i}=\sin \delta_{i} \cos \varphi-\cos \delta_{i} \cdot \sin \varphi \cdot \cos S_{i}^{*} \\
S_{i}=S_{i}^{*}-\lambda
\end{array}\right.
$$


After the completion of the sixth school, the static gyro enters the calibration phase, that is, the initial orientation error and random drift coefficient of the spindle of the electrostatic gyroscope in the inertial space are accurately measured. The initial orientation error is the difference between instantaneous measured value and instantaneous measured value, namely:

\subsection{8 hours calibration}

$$
\left\{\begin{array}{l}
\delta_{i}(t)=\tilde{\delta}_{i}^{P}(t)-\tilde{\delta}_{i}^{M}(t) \\
S_{i}(t)=\tilde{S}_{i}^{P}(t)-\tilde{S}_{i}^{M}(t)
\end{array}\right.
$$

When the height of the gyro on the electrostatic gyro monitor is less than hp, the filter and calibration time are reset. At present, TT \& C ship in the low latitude start electrostatic gyro monitor is generally the practice is to take a latitude outside the sailing strategy, while the static under the gyroscope when the initial value of the angle from the default value of $0^{\circ}$ to $5^{\circ}$, that $85^{\circ}$, which can avoid more than $87^{\circ}$ conditions. Timely adjust the course, so that the corresponding gyroscope height angle data curve smooth, using the upper and lower gyroscope "six school" time through the program.

\subsection{Floating start method proposed}

From the "six school" principle of work can be seen, gyroscope height angle and azimuth is the "six schools" in the process of two important factors in the calibration process, the need for high angle and azimuth angle to adjust Accuracy of Gyro Drift Parameters and Gyroscope Initial Position in Six - times Calibration. To ensure the accuracy of the initial position angle measurement, the system specifies the following constraints on h:

$$
\left\{\begin{array}{l}
\left|a h_{1}\right|>89.9945^{\circ} \\
\left|a h_{2}\right|>87^{\circ}
\end{array}\right.
$$

That is, the difference between the original measured value of the polar axis gyro height angle and the zero point of the altitude angle sensor is greater than the difference between the original measured value of the equatorial gyro height angle and the zero point of the elevation angle sensor is greater than 87 , Low-latitude region, it is easy to meet the constraints of more than $87^{\circ}$, resulting in six can not pass the normal calibration, on the other hand, if the ship wobbling, the replication error increases, the upper and lower gyro height angle error compensation value Too large, will lead to six schools can not pass.

After the completion of the sixth school, the static gyro enters the calibration phase, that is, the initial orientation error and random drift coefficient of the spindle of the electrostatic gyroscope in the inertial space are accurately measured.

The above analysis, the sea start gyro monitor, especially in low-latitude waters, due to the gyroscope's own performance degradation and bad sea conditions, easily lead to "six school" and 48 hours calibration can not pass, resulting in static can not be normal Into the navigation. Table 1 for a TT\&C ship in recent years, static low-latitude start operation.

At present, TT \& C ship in the low latitudes of the ship is usually used to maintain the dimension of navigation and other methods of static electricity, however, such as latitude sailing for the ship sailing conditions are more stringent requirements, by the navigation plan, the conditions of restricted sea area, In order to solve the above problems, this paper proposes a low-latitude drifting start method for the static gyro monitor.

\section{Drift start}

\subsection{Drift start conditions}

Electrostatic Gyro Monitor Six times during the school flight conditions is TT \& C ship uniform speed navigation to navigation, speed $9 \sim 12 \mathrm{kn}$, heading range of $\pm 1.5^{\circ}$, roll $<3^{\circ}$, pitch $<3^{\circ}$. Therefore, the drift start is generally selected in the distant sea conditions are better under the circumstances, when the current flow and flow rate is more stable, the ship's course, the location of the slow changes in attitude smooth.

2.2. Drift state characteristics 


\subsubsection{Heading and heading change rate}

In the drift state, the main factor affecting the course of the ship is the ocean current, which is affected by the surge and ocean current. As shown in Fig. 2 and Fig. 3, during one mission, the heading change of the electrostatic drift is about $20^{\circ} / 6 \mathrm{~h}$ and the maximum rate of heading change is no more than $5 \%$ s.

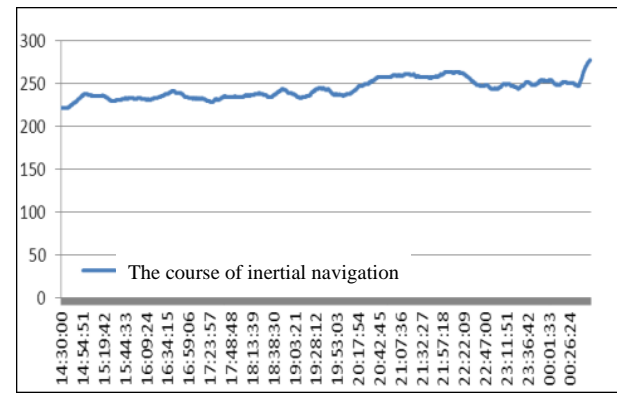

Figure 2. Variation of ship drift heading

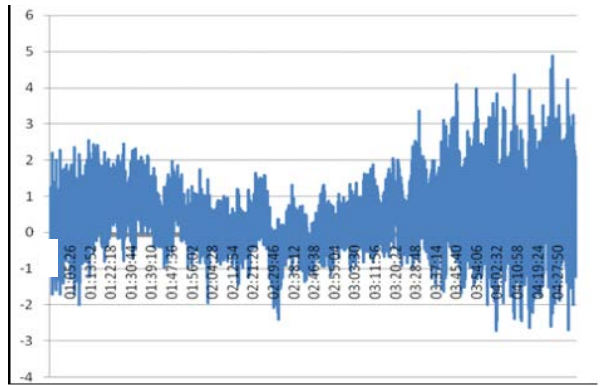

Figure 3. Variation rate of ship drift heading

2.2.2 Latitude and latitude rate of change

Figure 4 is a mission of the ship drift latitude changes and rate of change, can be seen from the figure, the ship drift, the latitude change does not exceed $0.02^{\circ} / 4 \mathrm{~h}$, the rate of change does not exceed $0.2 \mathrm{~m}$.

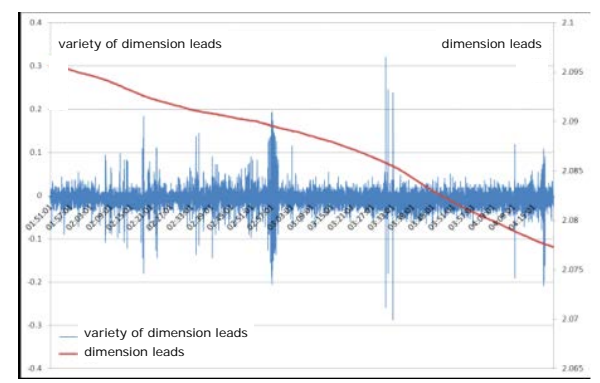

2.2.3 The ship is sloping

Figure 4. The rate of change of latitude and latitude of ship drift

Figure 5 for a mission ship horizontal, pitching situation, can be seen from the figure, the ship in the drift, the sea conditions in the better conditions, roll angle, pitch angle changes are very small, no more than $1.5^{\circ}$ The

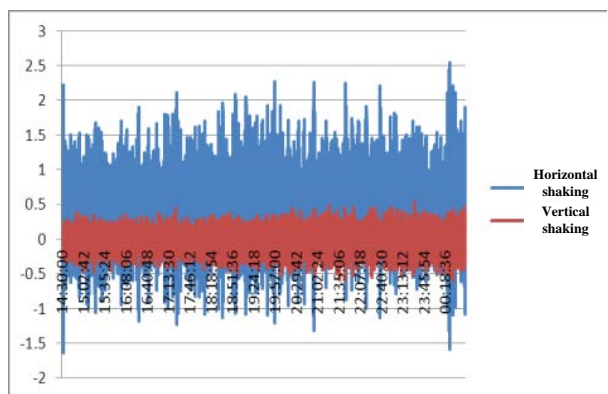

Figure 5. Ship drift horizontal, pitching situation

\section{Comparison and analysis of the start - up data of the drift and the latitude}

\section{$3.1 \mathrm{Z}$ vector}

The $\mathrm{Z}$ vector is the only observable amount in the static gyro monitor calibration process, as follows:

$$
\left\{\begin{array}{l}
Z_{1}=\Delta \delta_{1}+\Delta S_{1} \sin S_{2}-\Delta \delta_{1} \cos S_{1} \cos S_{2} \\
Z_{2}=\Delta \delta_{1} \cos S_{1} \cos \lambda+\Delta S_{1} \sin \lambda \\
Z_{3}=\Delta S_{2}+\left(\Delta S_{1} \cos \lambda-\Delta \delta_{1} \cos S_{1} \sin \lambda\right) \tan \Phi
\end{array}\right.
$$

$\mathrm{Z} 1$ is the error of the angle between the $\mathrm{H}$-axis of the polar axis gyroscope equatorial gyro, the position angle calculation error of the $\mathrm{Z} 2$ polar axis gyro $\mathrm{H}$ axis on the local meridian surface, and 
$\mathrm{Z} 3$ is the error of the position angle of the equatorial gyroscope on the equatorial plane. $\mathrm{Z}$ curve is an important indicator of static calibration. Ideally, the $\mathrm{Z}$ curve is 24 hours for the triangular function of the periodic change, as shown in Fig.

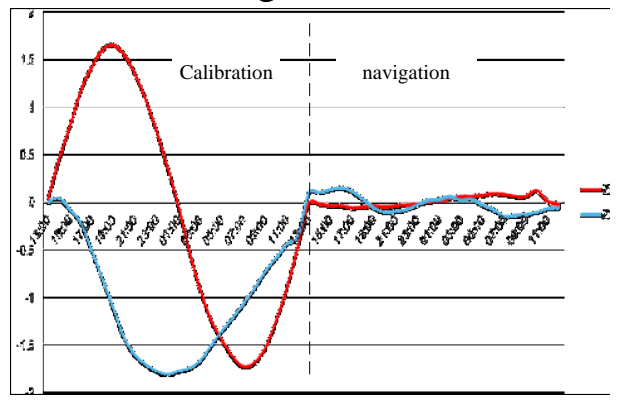

3.2 Static zero

Figure 6. Ideally, the electrostatic gyro monitor $\mathrm{Z}$ curve

Drift after the start of static zero stability, and the same latitude after the start of the zero-line consistency is better, in -0.2 to -0.6 angle points, as shown in Figure7, Figure 8.
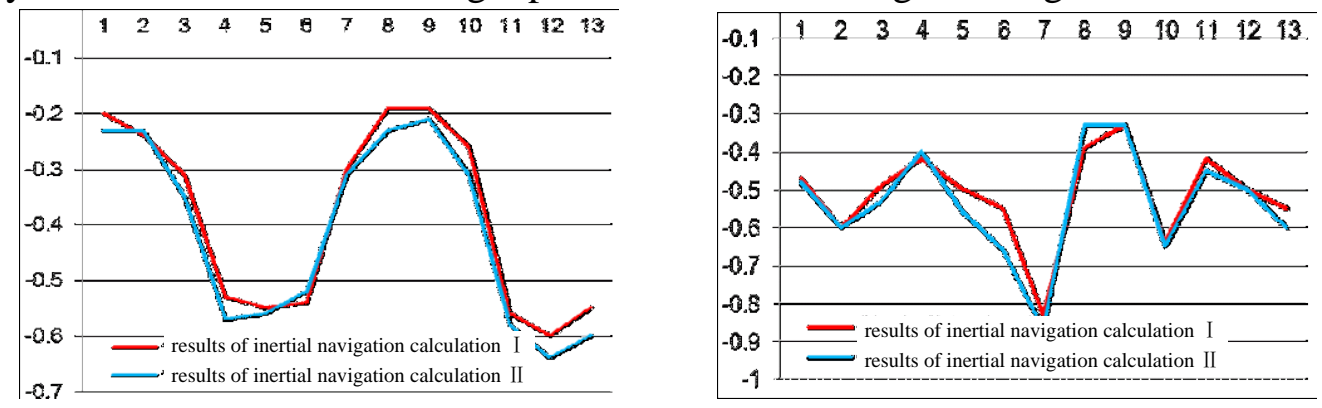

Figure 7.Wait for latitude to start static heading zero Figure 8.Drift start static heading zero

3.3 Contrast conclusion

In the case of better sea conditions (mainly refers to the impact of static six schools and calibration process in the process of change is relatively stable, and the ship location changes are relatively small, small boat), you can use drift start mode. Drift start position accuracy, heading accuracy, zero and other indicators and the same latitude sailing start quite accurate data to meet the requirements of indicators, can be combined with the ship navigation plan and energy saving and other factors to consider, flexible application.

\section{Summary}

The ship is in the drift state, because the latitude is basically unchanged, the ship vertical and horizontal are small, the course has regular changes and slow changes to meet the static gyro monitor start protection conditions, after the test verification, data accuracy to meet the requirements of the index, but also Can avoid a wide range of ship maneuver, save fuel for the successful operation of the electrostatic gyro monitor to provide a flexible use of the strategy.

\section{Reference}

[1] Liu Xinming, Sun Xuecheng. "Three times school" method of electrostatic gyro monitor [J]. Chinese Journal of Inertial Technology, 2011,19 (4): 0399-04.

Analysis of the relationship between the six - time calibration of the electrostatic gyro monitor and the azimuthal ring - like performance of the equatorial gyroscope. Chinese Journal of Inertial Technology, 2014,22 (3): 0281-03.

[3] Zhou Haiyuan, Ni Wenxiu. Comparison of several startup modes of electrostatic gyro monitor and its application analysis. Chinese Journal of Inertial Technology, 2015,23 (4): 0467-05. 